\title{
Miniaturization of MR Safe Pneumatic Rotational Stepper Motors
}

\author{
Vincent Groenhuis ${ }^{1}$ and Françoise J. Siepel ${ }^{1}$ and Stefano Stramigioli ${ }^{1,2}$
}

\begin{abstract}
Pneumatic rotational stepper motors can be used to actuate MR (magnetic resonance) safe robotic systems. This paper describes novel techniques to minimize the volumetric size and/or step size of such motors in order to cope with the limited space requirements while still delivering high precision. Three designs are presented: the $R-10$ measures $1.0 \mathrm{~cm}^{3}$, has step size $12.9^{\circ}$ and torque $1.2 \mathrm{~N} \mathrm{~mm}$. The $\mathrm{R}-40$ measures $25.6 \mathrm{~cm}^{3}$, has step size $1.01^{\circ}$ and torque $470 \mathrm{~N} \mathrm{~mm}$. The R-54 measures $46.7 \mathrm{~cm}^{3}$, has step size $1.01 \mathrm{~m}^{\circ}$ and torque $240 \mathrm{~N} \mathrm{~mm}$. The particularly small step size in the R-54 motor is achieved by using a high-reduction planetary gear.

These three motors demonstrate that small-scale rotational stepper motors with a wide range of step sizes and good torque characteristics can be constructed that surpass stateof-art designs by a considerable margin. This allows the advancement of MR safe robotics towards more compact and versatile designs, and also overcome certain existing limitations by combining multiple motors with different specifications.
\end{abstract}

\section{INTRODUCTION}

The MRI scanner offers high sensitivity compared to other three-dimensional imaging modalities without the adverse effects of ionizing radiation as in CT. Example applications are in periodic breast screening of high-risk patients, in addition to mammography and ultrasonography. When a suspicious lesion is found then it may need to be biopsied. If this lesion is only visible on MRI then an MRI-guided biopsy procedure is needed. Current manual procedures have drawbacks in terms of accuracy and speed [1], which have led to the research in MR safe and MR conditional robotic systems for breast biopsy.

MR safe robotic systems are highly preferred over MR conditional systems as the former utilizes the full potential of high-field MRI scanners with no restrictions. A major challenge in the development of any MR safe robotic system is the design of the actuator which needs to be free of metallic, ferromagnetic and conductive materials. The actuators must also be small enough to make the surgical robot fit inside the MRI scanner alongside the patient. With electromagnetic motors ruled out, pneumatics is one option which offers certain advantages over hydraulics, such as the small size and acceptability of small leakages [2], [3]. State-of-art pneumatic stepper motors have relative large dimensions (several centimetres) and/or step sizes (several degrees), so there is a need for smaller designs to actuate small-scale, high-precision MR safe robotic systems.

\footnotetext{
${ }^{1}$ V. Groenhuis, F.J. Siepel and S. Stramigioli are with Robotics and Mechatronics, University of Twente, Enschede, The Netherlands v.groenhuis@utwente.nl

${ }^{2}$ S. Stramigioli is with ITMO University, Saint Petersburg, Russia
}

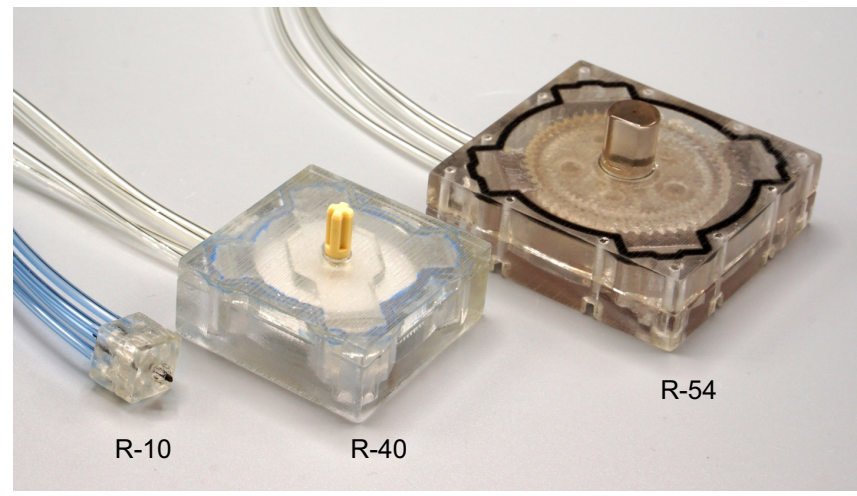

Fig. 1. The R-10, R-40 and R-54 pneumatic stepper motors.

\section{A. State of Art}

Several types of MR safe pneumatic stepper motors have been developed with a broad range of specifications. Stoianovici et al. developed the PneuStep with a build volume of $200 \mathrm{~cm}^{3}$, a step size of $3.3^{\circ}$ and a maximum specified torque of $600 \mathrm{Nmm}$ [4]. Guo et al. developed a pneumatic stepper motor with volume $84.7 \mathrm{~cm}^{3}$, step size $60^{\circ}$ and torque $4.9 \mathrm{Nmm}$ [5]. Sajima et al. developed a stepper motor with volume $28 \mathrm{~cm}^{3}$, step size $4.3^{\circ}$ and torque $170 \mathrm{Nmm}$ [6]. Farimani et al. developed the PneuAct with build size approx. $75 \mathrm{~cm}^{3}$ (including 1:40 gear), step size $3^{\circ}$ and torque $120 \mathrm{~N} \mathrm{~mm}$; a smaller version (without reduction gear) with build volume $4.6 \mathrm{~cm}^{3}$ and step size $120^{\circ}$ was also demonstrated [7]. Chen et al. developed a unidirectional stepper motor with build volume $4.7 \mathrm{~cm}^{3}$, step size $60^{\circ}$ and torque $2.4 \mathrm{~N} \mathrm{~mm} \mathrm{[8].}$

The authors of this paper previously presented several rotational stepper motors. The R-25 has a volume of $12.5 \mathrm{~cm}^{3}$, a step size of $6.9^{\circ}$ and a torque of $100 \mathrm{~N} \mathrm{~mm}$ [9]. A dualspeed version, the DR-32, was also demonstrated with a build volume of $22.6 \mathrm{~cm}^{3}$, differential step size $2.9^{\circ}$ and torque $74 \mathrm{~N} \mathrm{~mm} \mathrm{[10].}$

MR safe surgical robots powered by pneumatic stepper motors have also been demonstrated. The Sunram 5 and previous iterations from the authors of this paper are driven by combinations of linear and curved stepper motors [11], [12], while pneumatic robotic systems by other authors are typically driven by rotational or helical stepper motors [13], [14].

Fig. 11 graphically shows the build volumes and step sizes of the state-of-art motors in blue. It shows that these all have build volumes over $4 \mathrm{~cm}^{3}$ and step sizes higher than $2^{\circ}$. The smallest motors (in volume) are not directly 


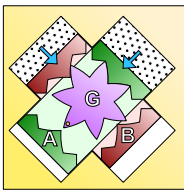

0

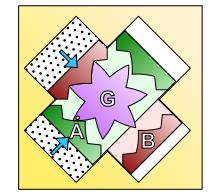

1

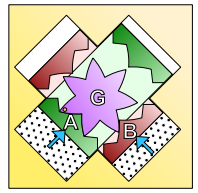

2

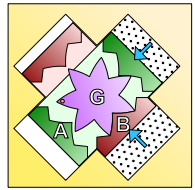

3
Fig. 2. Sequence of piston states during clockwise rotation of the gear. After four steps the motor is back in the first state, but with the gear displaced by one tooth.

usable due to the unidirectional mechanism (Chen) or lack of torque (Farimani). Bidirectional stepper motors with sufficient torque have relatively large dimensions, step sizes and/or a complicated design which limit the applicability in small-scale MR safe robotics. There is a need for new designs that have smaller dimensions and/or step sizes than state-of-art designs, hereby advancing the Pareto frontier in Fig. 11 towards the left and bottom edges of the graph.

This paper is organized as follows. First the working principles, design and construction of the R-10, R-40 and R-54 are described. Next, torque measurements and step size validation experiments are described alongside with the results. Finally, conclusions are drawn with an outlook of application in future MR safe robotic systems.

\section{MATERIALS AND METHODS}

Three different rotational stepper motors are presented. All three models make use of two pneumatic cylinders with rectangular cross-section, positioned in a cross-shape that interact with a gear. Several aspects of this type of pneumatic stepper motor have been extensively described and investigated by Groenhuis et al. [9]. The state sequence is shown in Fig. 2: by applying pressure in the four chambers in the given sequence a step-wise rotational motion is generated.

The R-10 is optimized for minimal volume by making the cylinders and gears as small as practically feasible by rapid prototyping without taking step size or torque into account. In contrast, the R-54 is optimized for minimal step size by integrating a high-reduction planetary gear transmission. Finally, the R-40 is designed for minimal volume while keeping the step size fixed at approximately $1.0^{\circ}$.

\section{A. R-10 design}

The $\mathrm{R}-10$ is a scaled-down version of the R-25. It consists of the housing with cover, two pistons with four silicone seals, one gear with seven teeth and the axle. The dimensions of the housing are $(10 \times 10 \times 10) \mathrm{mm}^{3}=1.0 \mathrm{~cm}^{3}$. Fig. 3 shows a CAD drawing of the motor.

The cylinder cross-sectional area measures $(3 \times 4) \mathrm{mm}^{2}$ $=12 \mathrm{~mm}^{2}$. The gear has an outside diameter of $4 \mathrm{~mm}$, the seven teeth have a depth of $1 \mathrm{~mm}$. The step size is $\frac{90}{7}=$ $12.9^{\circ}=0.224 \mathrm{rad}$. At a system pressure of $0.1 \mathrm{MPa}$ (approx. 1 bar) the piston force is $12 \cdot 10^{-6} \mathrm{~m}^{2} \cdot 0.1 \cdot 10^{6} \mathrm{~N} / \mathrm{m}^{2}=1.2 \mathrm{~N}$. The nominal work per step is $\frac{1}{2} \cdot 1 \mathrm{~mm} \cdot 1.2 \mathrm{~N}=0.6 \mathrm{~mJ}$, resulting in a nominal torque of $\frac{0.6 \mathrm{~mJ}}{0.224 \mathrm{rad}}=2.68 \mathrm{~N} \mathrm{~mm}$ for every $0.1 \mathrm{MPa}$ of pressure, equivalent to $26.8 \mathrm{Nmm} / \mathrm{MPa}$.

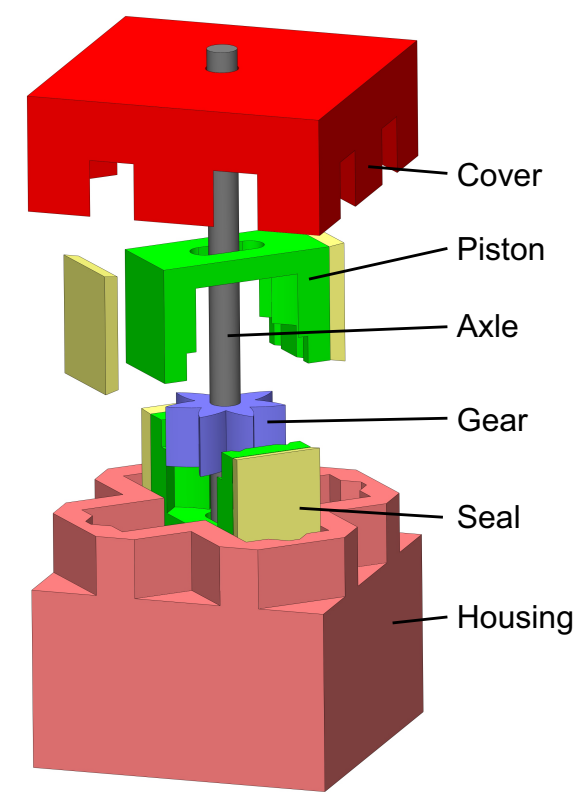

Fig. 3. R-10 CAD drawing, showing the housing with cover, axle, gear, two pistons and four seals. The pneumatic tube sockets are on the bottom of the housing and not visible.

\section{B. $R-40$ design}

The R-40 is also based on the R-25 design and consists of the same type of components as the R-10. The main difference is that the gear has 89 teeth, resulting in a step size of $\frac{90}{89}=1.0112^{\circ}$. Given the minimum teeth tip spacing of $1.0 \mathrm{~mm}$ that can still be rapid prototyped without compromising on torque and reliability, the 89-teeth gear was designed to have an outside diameter of $30 \mathrm{~mm}$ and a teeth depth of $1.2 \mathrm{~mm}$. The external dimensions are $(40 \mathrm{x} 40 \mathrm{x}$ 16) $\mathrm{mm}^{3}=25.6 \mathrm{~cm}^{3}$. Fig. 4 shows a CAD drawing of the R-40 motor.

The cylinders of the $\mathrm{R}-40$ have a cross-sectional area of $(10 \times 10) \mathrm{mm}^{2}$. Combined with an effective stroke of $0.6 \mathrm{~mm}$, for every $0.1 \mathrm{MPa}$ of pressure this results in 100 . $10^{-6} \mathrm{~m}^{2} \cdot 0.1 \cdot 10^{6} \mathrm{~N} / \mathrm{m}^{2} \cdot 0.6 \mathrm{~mm}=6.0 \mathrm{~mJ}$ of energy delivered per step. With a step angle of $1.0112^{\circ}=0.0176 \mathrm{rad}$ this corresponds to a theoretical torque of $\frac{6.0 \mathrm{~mJ}}{0.0176 \mathrm{rad}}=341 \mathrm{~N} \mathrm{~mm}$ for every $0.1 \mathrm{MPa}$ of pressure, equivalent to $3.41 \mathrm{Nm} / \mathrm{MPa}$.

\section{C. $R-54$ design}

The R-54 is different from the other two described motors in the sense that it includes a planetary gear, allowing for a significant reduction in step size. Fig. 5 shows a CAD drawing of the R-54 motor.

The kinematic diagram is shown in Fig. 6. The pneumatic cylinders (1) are attached to the housing and operate the pistons (2). The different teeth on the pistons engage with both the carrier (3) and the ring gear (4), resulting in step motions over different angles. The shafts of the planet gears (5) are mounted on the carrier (3) while the planet gears themselves are driven by the ring gear (4). Finally, the sun gear (6) is driven by the planet gears (5) and attached to the output shaft. 


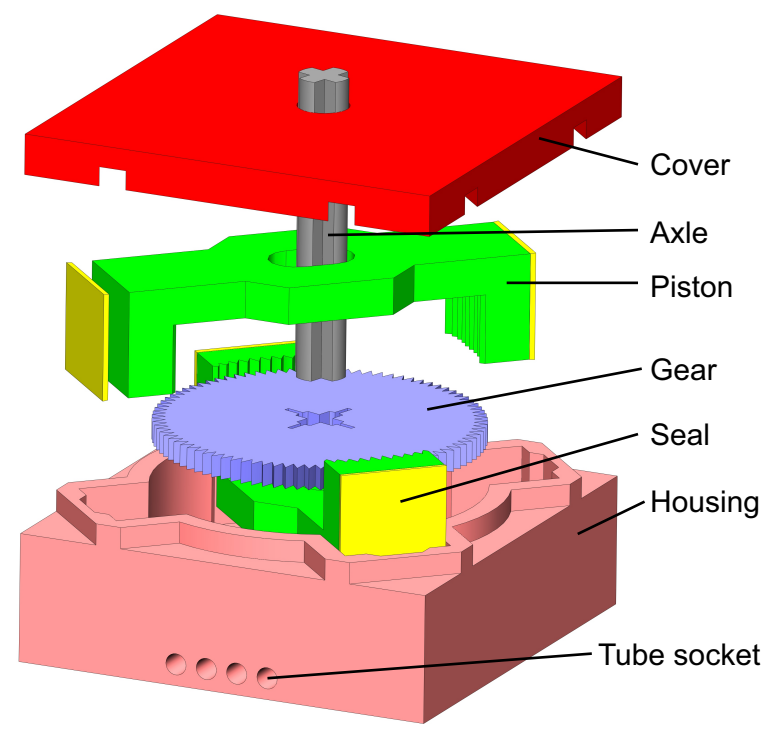

Fig. 4. R-40 CAD drawing, showing exactly the same type components as the R-10 but with different dimensions and a higher number of teeth on the gear.

The step sizes of the different bodies depend on te number of teeth on its perimeters. Let $T_{s}, T_{r}$ and $T_{p}$ be the teeth count of the sun, internal ring and planet gear, respectively. Likewise, let $T_{e}$ and $T_{c}$ be the teeth count of the (pneumatically actuated) external ring and carrier gears. Finally, let $S_{s}, S_{r}$ and $S_{c}$ be the step sizes (in degrees) of the sun, ring and carrier gears, respectively.

The goal is to find a suitable assignment of $\left(T_{s}, T_{p}, T_{r}, T_{c}, T_{e}\right)$ such that $S_{s}$ is minimal. For this we have to describe $S_{s}$ as function of the teeth parameters.

First, we calculate the step sizes $S_{r}$ and $S_{c}$ of the pneumatically actuated ring and carrier gears. As shown in Fig. 2, four steps advance the gear by exactly one teeth:

$$
S_{r}=\frac{360}{4 T_{e}}=\frac{90}{T_{e}}, S_{c}=\frac{90}{T_{c}}
$$

Next, we apply the planetary gear equations. The planet gears are constrained by the sun gear and internal ring gear:

$$
\begin{aligned}
T_{r} & =T_{s}+2 T_{p} \\
S_{s} & =-\frac{T_{r}}{T_{s}}\left(S_{r}-S_{c}\right)+S_{c}
\end{aligned}
$$

We use the following parameter constraints to take account for rapid prototyping ability, proper gear meshing and keeping dimensions sufficiently small:

$$
\begin{aligned}
16 & \leq T_{s}, T_{p} \leq 25 \\
T_{e}, T_{c} & \leq 81 \\
T_{e}, T_{c} & \equiv 1 \quad(\bmod 4)
\end{aligned}
$$

The latter constraint simplifies piston design as the piston teeth of all four sides can be shaped equally.

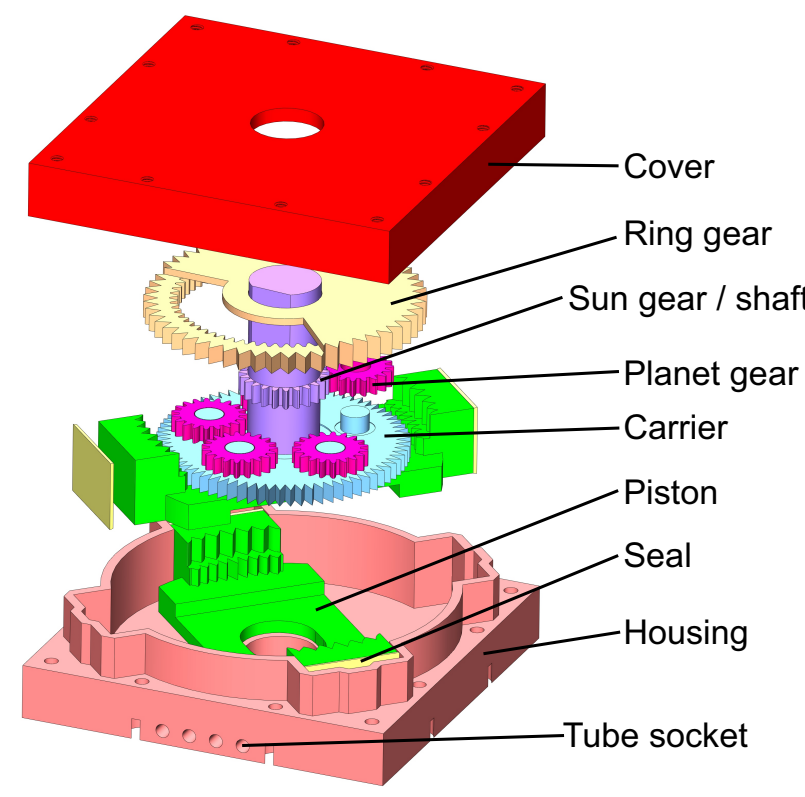

Fig. 5. R-54 CAD drawing. The housing and pneumatics are similar to that of the R-10 and R-40, but the single gear is replaced by a planetary gear mechanism consisting of the ring gear, sun gear, carrier and planet gears.

The two pistons engage on both the carrier gear and the ring gear.

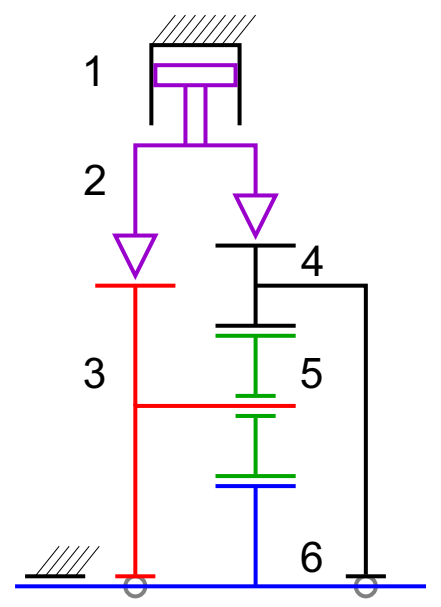

Fig. 6. Kinematic diagram of the R-54 showing the pneumatic cylinder (1), piston with teeth (2), carrier (3), ring gear (4), planet gear (5) and sun gear on output shaft (6).

The free parameters are $T_{s}, T_{p}, T_{e}$ and $T_{c}$ and the optimization criterion is $\min \left|S_{s}\right|$. The state space size is $10^{2} \cdot 20^{2}=40000$, which is small enough to perform an exhaustive search over all possible parameter combinations. The optimum has the following values:

$$
\left(T_{s}, T_{p}, T_{r}, T_{c}, T_{e}\right)=(23,19,61,73,53)
$$

Filling these values in Eq. 3 gives: 


$$
\begin{aligned}
S_{s} & =-\frac{T_{r}}{T_{s}}\left(\frac{90}{T_{e}}-\frac{90}{T_{c}}\right)+\frac{90}{T_{c}} \\
& =90 \cdot \frac{T_{s} T_{e}+T_{r} T_{e}-T_{r} T_{c}}{T_{s} T_{e} T_{c}} \\
& =90 \cdot \frac{23 \cdot 53+61 \cdot 53-61 \cdot 73}{23 \cdot 53 \cdot 73} \\
& =90 \cdot \frac{-1}{88987}=-0.0010114^{\circ}=-1.0114 \mathrm{~m}^{\circ}
\end{aligned}
$$

For the planetary gears we use gear modulus of 0.5 which corresponds to a teeth spacing of $\frac{\pi}{2}=1.6 \mathrm{~mm}$. The outer diameter of the external ring gear is set to $39 \mathrm{~mm}$ with a teeth depth of $2 \mathrm{~mm}$ to keep sufficient margin (approx. $1.5 \mathrm{~mm}$ ) to the internal ring gear. The carriage gear has more teeth $\left(T_{c}=73\right)$ than the external ring gear $\left(T_{e}=53\right)$, but the teeth spacing is still large enough to allow a slightly smaller outer diameter of $35 \mathrm{~mm}$. This smaller diameter makes it easier to construct the piston teeth by stacking different rows of teeth without need for supports.

The pneumatic cylinders have a cross-sectional area of $(14 \times 10) \mathrm{mm}^{2}=140 \mathrm{~mm}^{2}$, giving $14 \mathrm{~N}$ of force per $0.1 \mathrm{MPa}$ of pressure. The teeth depth is $2 \mathrm{~mm}$, so every $0.1 \mathrm{MPa}$ of pressure results in $14 \mathrm{~N} \cdot \frac{1}{2} \cdot 2 \mathrm{~mm}=14 \mathrm{~mJ}$ of energy delivered per step.

There are two parallel drivetrains from the pneumatic cylinders to the output shaft. As the carrier and ring gears are not coupled together, overloading either of them will result in skipped steps. It is therefore required to evaluate each drivetrain separately in determining the theoretical torque on the output shaft.

- Ring gear: The step size is $\frac{90}{53} \approx 1.70^{\circ} \approx 0.030 \mathrm{rad}$ and this corresponds to a theoretical torque on the ring of $\frac{14 \mathrm{~mJ}}{0.030 \mathrm{rad}} \approx 472 \mathrm{Nmm}$ for every $0.1 \mathrm{MPa}$ of pressure, equivalent to $4.72 \mathrm{Nm} / \mathrm{MPa}$. The torque ratio from ring to sun is $\left|-\frac{T_{s}}{T_{r}}\right|=\frac{23}{61} \approx 0.38$, so the theoretical output torque (through the ring) is $\frac{23}{61} \cdot 4.72 \mathrm{Nm} / \mathrm{MPa} \approx$ $1.78 \mathrm{Nm} / \mathrm{MPa}$.

- Carrier gear: The step size is $\frac{90}{73} \approx 1.23^{\circ} \approx 0.022 \mathrm{rad}$ and this corresponds to a theoretical torque on the carrier of $\frac{14 \mathrm{~mJ}}{0.022 \mathrm{rad}} \approx 651 \mathrm{~N} \mathrm{~mm}$ for every $0.1 \mathrm{MPa}$ of pressure, equivalent to $6.51 \mathrm{Nm} / \mathrm{MPa}$. The torque ratio from carrier to sun is $\left|\frac{1}{1+T_{r} / T_{s}}\right|=\frac{T_{s}}{T_{r}+T_{s}}=\frac{23}{84} \approx 0.27$, so the theoretical output torque (through the ring) is $\frac{23}{84} \cdot 6.51 \mathrm{Nm} / \mathrm{MPa} \approx 1.78 \mathrm{Nm} / \mathrm{MPa}$.

The theoretical output torques for both drivetrains are equal (up to a rounding error), which is the consequence of having almost equal step sizes in opposite directions and equal teeth depth in the carrier gear and external ring gear. The small difference between the step sizes account for the $1.01 \mathrm{~m}^{\circ}$ net step size. When the output shaft is loaded by some torque, this torque propagated through both drivetrains independent of the torque direction and rotational motion. As the transferred output torque through both drivetrains is approximately equal, we can define the theoretical output torque of the R-54 motor as $1.78 \mathrm{Nm} / \mathrm{MPa}$.

\section{Fabrication}

The majority of the parts was 3-D printed on an Objet Connex3 (Stratasys, Eden Prairie, MN, USA) in VeroClear material in standard quality, glossy surface mode. The housing cover in the R-54 also includes an integrated stationary seal printed in TangoBlack. The gears in the R-10 and R40 motors were laser-cut from $2 \mathrm{~mm}$ acetal. The axle in the R-10 was cut from a $1 \mathrm{~mm}$ diameter carbon fibre rod and the axle in the R-40 consists of a Lego Technic axle. The moving seals were laser-cut from a $0.5 \mathrm{~mm}$ thick silicone rubber sheet with an approximate stiffness of Shore A 60.

After assembling the motor parts the cover was glued on the housing using cyanoacrylate (Loctite $406+770$ ). This glue was also used to fix the four pneumatic tubes $(2 \mathrm{~mm}$ outer diameter) in the sockets and to fix the gear in the R-10 to the axle. The assembled motors are shown in Fig. 1.

\section{E. MR safety analysis}

The three motors consist of the following materials:

- VeroClear: all motors (housing, pistons, planetary gears)

- TangoBlack: R-54 (stationary seal)

- Acetal (POM): R-10 and R-40 (gears)

- Carbon fibre: R-10 (axle)

- ABS: R-40 (axle)

- Silicone rubber: all motors (moving seals)

- Polyurethane: all motors (tubing)

- Glue (Loctite 406, 770): all motors

- Sealant (Loctite 5926): R-40

All listed materials are non-metallic, non-ferromagnetic and non-conductive, with one exception: carbon fibre is conductive. This implies that only the R-40 and R-54 are classified MR safe according to the ASTM F2503 standard. The R-10 may be classified MR safe when the carbon fibre axle is replaced by a different material.

\section{EXPERIMENTAL RESULTS AND ANALYSIS}

The three motors were characterized by measuring the torque-pressure relationship. Also, the actual step sizes were validated by observation.

\section{A. Measurement setup}

For torque measurements the setup shown in Fig. 7 is used. The motor is mounted on the table with a frame and a winch is placed on the motor shaft. This winches a fishing line over a pulley, lifting up one or more weights of known mass. The radius of the winch is $3.5 \mathrm{~mm}$ for the $\mathrm{R}-10$ motor and $24 \mathrm{~mm}$ for the R-40 and R-54 motors. The total mass is varied and for each given mass a measurement point is collected. The measurement involves slowly increasing the system pressure using a pressure regulator until the motor can consistently lift the given mass. This procedure is repeated to obtain at least five data points. The data points for each motor are then plotted in a pressure-torque graph.

The step size validation involves counting the number of steps in one full revolution and comparing these to the calculated values. For the R-10 the theoretical number of steps is 28 , for the R-40 it is 356 steps and for the R-54 it is 


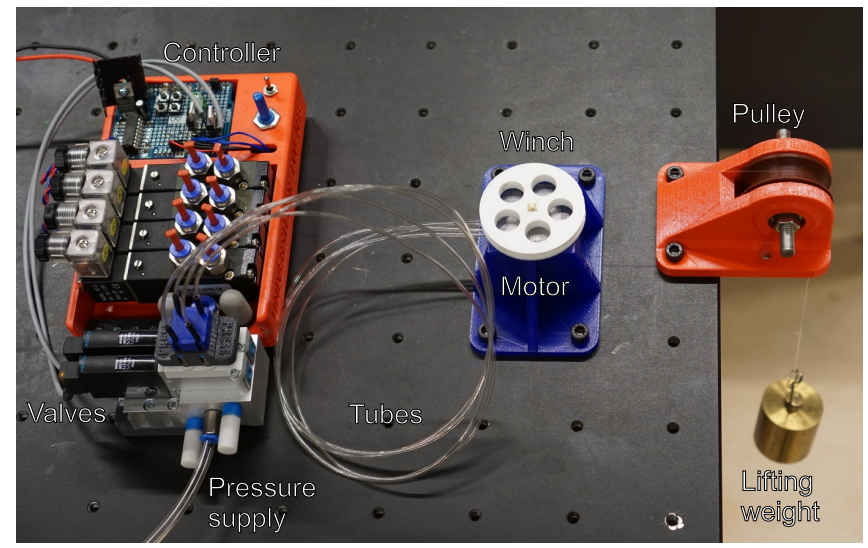

Fig. 7. Setup for torque measurements. The valves are controlled by an Arduino controller, sending pressure waveforms to the motor through the four tubes. The motor then lifts a weight of known mass over a pulley by means of a winch.

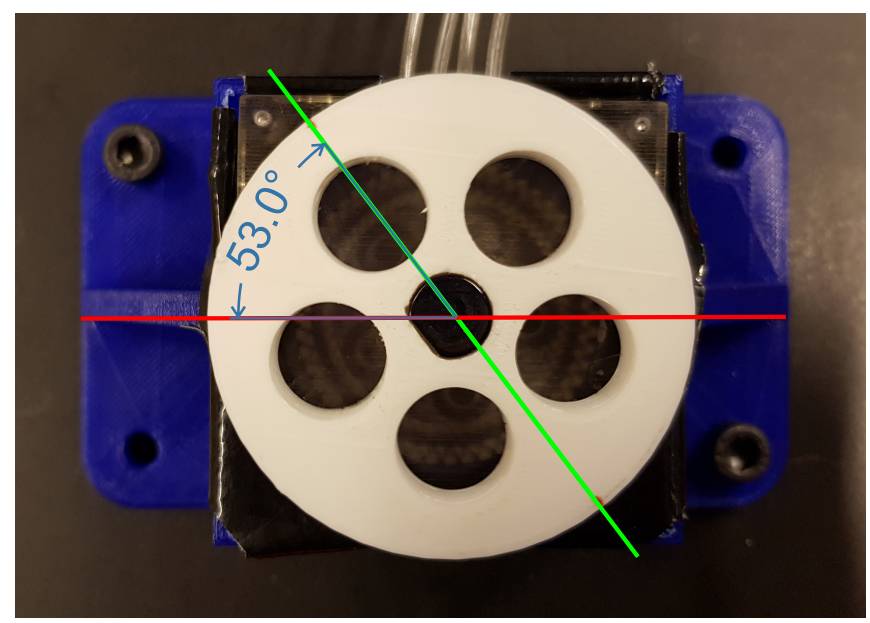

Fig. 8. Setup for angle measurements on the R-54 motor.

355948 steps. Validating these numbers are trivial for the R10 and R-40. For the R-54, validation is done by running the motor at a fixed stepping frequency of $99 \mathrm{~Hz}$ and observing the angle of the output shaft pulley at different moments using a camera. The observed angular velocity can then be compared to the predicted angular velocity as calculated from the step angle and stepping frequency.

\section{B. Measurement results}

1) Torque measurement results: Fig. 9 shows the torque graph of the R-10, R-40 and R-54 motors. The maximum torque of the $\mathrm{R}-10$ was measured to be $1.2 \mathrm{Nmm}$ at a pressure of $0.4 \mathrm{MPa}$. The graph is approximately linear, with a slope of $3.81 \cdot 10^{-9} \mathrm{~N} \mathrm{~Pa}^{-1}$ or $3.81 \mathrm{Nmm} / \mathrm{MPa}$. When compared to the theoretical torque of $26.8 \mathrm{Nmm} / \mathrm{MPa}$, the mechanical efficiency is $\frac{3.81}{26.8}=14 \%$.

The maximum torque of the R-40 was measured to be $470 \mathrm{Nmm}$ at a pressure of $0.26 \mathrm{MPa}$. This graph is also approximately linear, with a slope of $2.03 \mathrm{Nm} / \mathrm{MPa}$. When compared to the theoretical torque of $3.41 \mathrm{Nm} / \mathrm{MPa}$, the mechanical efficiency is $\frac{2.03}{3.41}=60 \%$.

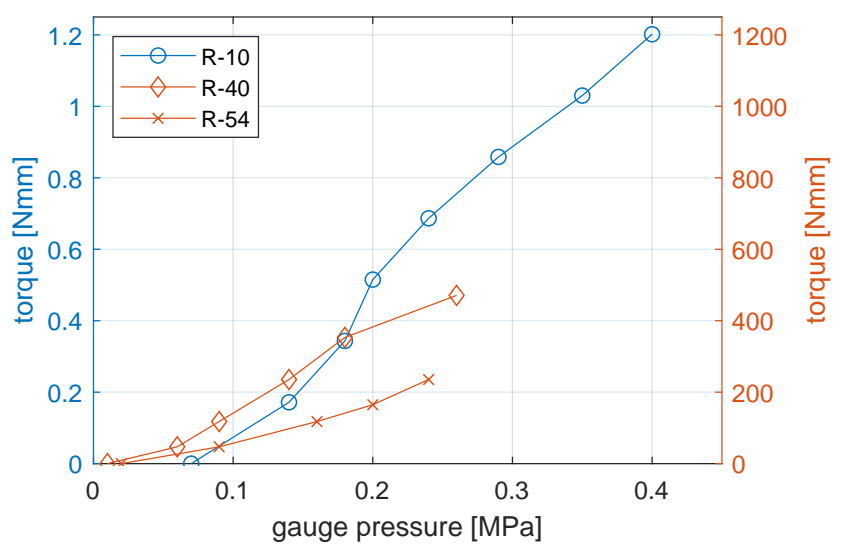

Fig. 9. Torque versus pressure diagrams for the three motors.

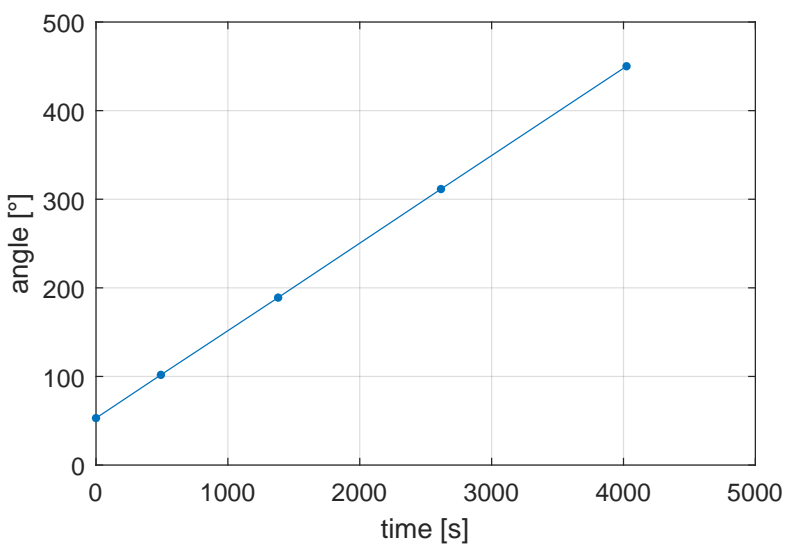

Fig. 10. Observed angle of the R-54 as function of elapsed time.

The R-54 was measured to have a maximum torque of $240 \mathrm{Nmm}$ at a pressure of $0.24 \mathrm{MPa}$. Again, this graph is approximately linear and the slope is $1.05 \mathrm{Nm} / \mathrm{MPa}$. When compared to the theoretical torque of $1.78 \mathrm{Nm} / \mathrm{MPa}$, the mechanical efficiency is $\frac{1.05}{1.78}=59 \%$.

2) Step size validation results: The number of steps per revolution of the R-10 and R-40 were counted and found to be equal to 28 and 356 , respectively, which are in accordance with the theoretical values.

The R-54 was left running for one hour at a stepping frequency of $99 \mathrm{~Hz}$. At five instances a photo was taken of the output shaft from which the angle was extracted using graphics software. These angles are plotted in Fig. 10. The angular velocity was measured to be $0.0987^{\circ} / \mathrm{s}$, at a stepping frequency of $99 \mathrm{~Hz}$ this is equivalent to a step size of $0.997 \mathrm{~m}^{\circ}$ and consistent with the theoretical value of $1.011 \mathrm{~m}^{\circ}$, up to the measurement error.

\section{Comparison and Discussion}

Table I lists the specifications and measured characteristics of the R-10, R-40 and R-54 motors. Fig. 11 graphically shows the build sizes and step sizes of the R-10, R-40 and R54 in red so that these dimensions can be directly compared to state-of-art motors. 
TABLE I

COMPARISON OF R-10, R-40 AND R-54 PNEUMATIC STEPPER MOTORS.

\begin{tabular}{c|c|c|c|c|c|c} 
Motor & Dimensions & Step size & Theoretical torque & Effective torque & Efficiency & Max. power \\
\hline R-10 & $10 \times 10 \times 10 \mathrm{~mm}^{3}=1.0 \mathrm{~cm}^{3}$ & $12.9^{\circ}$ & $27 \mathrm{Nmm} / \mathrm{MPa}$ & $3.8 \mathrm{Nmm} / \mathrm{MPa}$ & $14 \%$ & $40 \mathrm{~mW}$ \\
R-40 & $40 \times 40 \times 16 \mathrm{~mm}^{3}=26 \mathrm{~cm}^{3}$ & $1.01^{\circ}$ & $3.4 \mathrm{Nm} / \mathrm{MPa}$ & $2.0 \mathrm{Nm} / \mathrm{MPa}$ & $60 \%$ & $800 \mathrm{~mW}$ \\
R-54 & $54 \times 54 \times 16 \mathrm{~mm}^{3}=47 \mathrm{~cm}^{3}$ & $1.01 \mathrm{~m}^{\circ}$ & $1.8 \mathrm{Nm} / \mathrm{MPa}$ & $1.1 \mathrm{Nm} / \mathrm{MPa}$ & $59 \%$ & $4.2 \mathrm{~mW}$
\end{tabular}

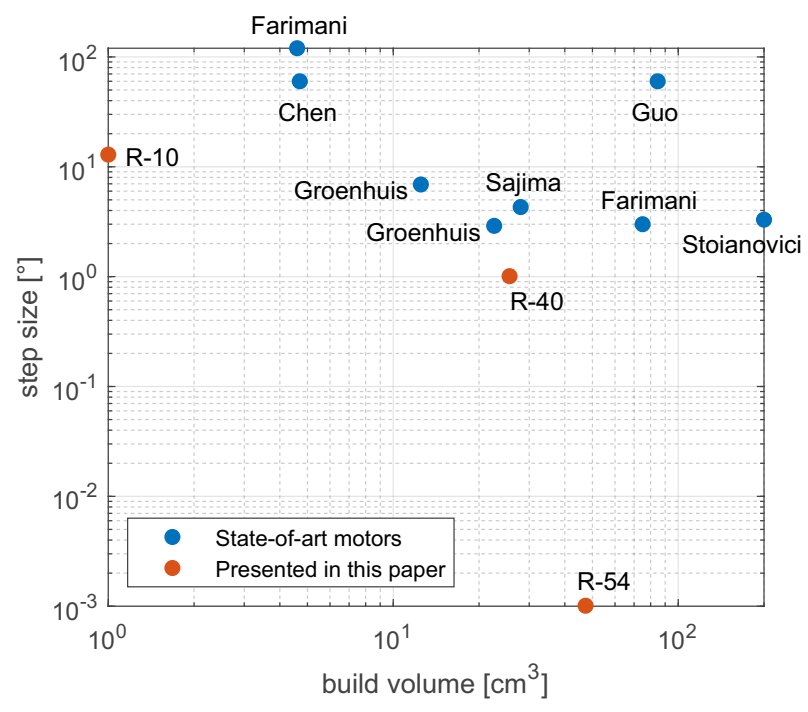

Fig. 11. Graphical comparison of build volumes and step sizes of stateof-art motors (blue) and the three motors presented in this paper (red).

The R-10 is smaller than any other motor by a considerable margin and this shows that it is possible to construct a miniature MR safe pneumatic stepper motor measuring only $10 \mathrm{~mm}$ in every direction (excluding axle). The measured torque of $1.2 \mathrm{~N} \mathrm{~mm}$ is relatively low. The mechanical efficiency is only $14 \%$ which can be attributed to several factors. The finite 3-D printing resolution results in blunt teeth tips in the pistons resulting in a suboptimal wedge mechanism [10]. Another important factor is the high perimeter-to-surface ratio which causes relatively high friction in the moving parts, reducing the effective force of the cylinders. Still, a further reduction in size may be possible by optimizing the design and production methods, but reduction gears will be needed in practical applications.

The R-40 motor combines a practical step size and adequate torque in a housing which is still compact enough for use in MR safe surgical robotics. The build volume is comparable to that of several state-of-art designs, but the step size of $1.01^{\circ}$ is smaller than any of these. The limiting factor here is the minimum gear teeth tip spacing needed for reliable meshing with the piston teeth taking the limitations of rapid prototyping into account.

The R-54 shows an extreme example of step size reduction by effectively exploiting the capabilities of the planetary gear. While the demonstrated step size of $1.01 \mathrm{~m}^{\circ}$ is too small to have any practical value in MR safe robotics, the step size equation shows that by carefully choosing the right gear sizes virtually any step size can be obtained. One application requiring relatively small step sizes (order of $0.1^{\circ}$ ) are steerable catheters for endovascular applications. The build volume of the R-54 is larger than that of the R-40, but there is sufficient room for miniaturization. The planetary gear modulus could be reduced from 0.5 down to 0.33 and also the number of teeth on the external ring and carrier gears could be reduced, resulting in a stepper motor smaller than the R-40 but with broad customizability of step sizes.

The motors were tested with relatively short pneumatic tubes. In MR safe applications the valve manifold is typically placed outside the Faraday cage of the MRI scanner, requiring long $(5 \mathrm{~m})$ tubes between motors and manifold. This reduces the maximum stepping frequency to approx. $8 \mathrm{~Hz}$ without compromising on torque [9]; higher stepping frequencies cause a reduction in output torque due to the pneumatic transmission line characteristics.

The wedge mechanism involves sliding contact surfaces which are subject to friction losses and wear. This is mitigated by the use of low-friciton materials (acetal and glossyprinted VeroClear), the application of a suitable lubricant (petroleum jelly) and by involving multiple teeth per contact to better distribute the load. It has been shown that the amount of wear in certain designs is negligible [9] and the friction and wear can be further reduced by optimizing the material choice.

The R-40 and R-54 are inherently MR safe. The presented R-10 first needs to have its carbon fibre axle replaced by e.g. a fibreglass rod of the same diameter before it can also be classified MR safe.

\section{CONCLUSION}

The three motors presented in this paper show that significant reduction in build volume and/or step size in MR safe pneumatic stepper motors is possible compared to state-ofart stepper motors. The R-10 only measures $10 \mathrm{~mm}$ in every direction, the R-40 offers a good compromise between build dimensions and step size and the R-54 demonstrates a very small step size of $1.0 \mathrm{~m}^{\circ}$ due to the integration of a highreduction planetary gear.

The described developments offer several possibilities in future MR safe robotics. Small individual motors with different characteristics can be coupled to form dual-speed motors on every axis, hereby circumventing the low bandwidth associated with long pneumatic tubes. Miniature motors such as the R-10 could be integrated in the tip of an endoscope and offer a pneumatic alternative to conventional wire actuation techniques. Lastly, the planetary gearbox design in the R-54 
enables customization of the step size tailored to the specific application.

\section{REFERENCES}

[1] J. Veltman, C. Boetes, T. Wobbes, J. G. Blickman, and J. O. Barentsz, "Magnetic resonance-guided biopsies and localizations of the breast: initial experiences using an open breast coil and compatible intervention device," Investigative radiology, vol. 40, no. 6, pp. 379-384, 2005.

[2] Y. Chen, I. Godage, H. Su, et al. "Stereotactic Systems for MRI-Guided Neurosurgeries: A State-of-the-Art Review," Annals of Biomedical Engineering, vol. 47, no. 2, pp. 335-353, Feb. 2019. doi: 10.1007/s10439-018-02158-0

[3] R. Monfaredi, K. Cleary, and K. Sharma, "MRI Robots for NeedleBased Interventions: Systems and Technology," Annals of Biomedical Engineering, vol. 46, no. 10, pp. 1479-1497, 2018.

[4] D. Stoianovici, A. Patriciu, D. Petrisor, D. Mazilu and L. Kavoussi, "A New Type of Motor: Pneumatic Step Motor," in IEEE/ASME Transactions on Mechatronics, vol. 12, no. 1, pp. 98-106, Feb. 2007. doi: 10.1109/TMECH.2006.886258

[5] Z. Guo, T.T.L. Lun, Y. Chen, H. Su, D.T.M. Chan, K.W. Kwok, "Novel Design of an MR-safe Pneumatic Stepper Motor for MRIguided Robotic Interventions," Proceedings of 9th Hamlyn Symposium on Medical Robotics, London, United Kingdom, pp. 50-51, June 2016.

[6] D. Stoianovici, A. Patriciu, D. Petrisor, D. Mazilu and L. Kavoussi, "A New Type of Motor: Pneumatic Step Motor," in IEEE/ASME Transactions on Mechatronics, vol. 12, no. 1, pp. 98-106, Feb. 2007.

[7] F. S. Farimani and S. Misra, "Introducing PneuAct: ParametricallyDesigned MRI-Compatible Pneumatic Stepper Actuator," 2018 IEEE International Conference on Robotics and Automation (ICRA), Brisbane, QLD, 2018, pp. 200-205. doi: 10.1109/ICRA.2018.8462697
[8] Y. Chen, C. D. Mershon and Z. T. H. Tse, "A 10-mm MR-Conditional Unidirectional Pneumatic Stepper Motor," in IEEE/ASME Transactions on Mechatronics, vol. 20, no. 2, pp. 782-788, April 2015. doi: 10.1109/TMECH.2014.2305839

[9] V. Groenhuis and S. Stramigioli, "Rapid Prototyping HighPerformance MR Safe Pneumatic Stepper Motors," in IEEE/ASME Transactions on Mechatronics, vol. 23, no. 4, pp. 1843-1853, Aug. 2018. doi: 10.1109/TMECH.2018.2840682

[10] V. Groenhuis, F.J. Siepel and Stramigioli, "Dual-Speed MR Safe Pneumatic Stepper Motors," Robotics: Science and Systems, Pittsburgh, United States, June 26-30, 2018.

[11] V. Groenhuis, F.J. Siepel, M.K. Welleweerd, J. Veltman and S. Stramigioli, "Sunram 5: An MR Safe Robotic System for Breast Biopsy." Proceedings of Hamlyn Symposium on Medical Robotics, London, United Kingdom, pp. 82-83, June 2018.

[12] V. Groenhuis, F.J. Siepel, J. Veltman, J.K. van Zandwijk, S. Stramigioli, "Stormram 4: An MR Safe Robotic System for Breast Biopsy." Annals of Biomedical Engineering, vol. 46, no. 10, pp. 1686-1696, 2018. doi: 10.1007/s10439-018-2051-5

[13] D. Stoianovici et al., "Multi-Imager Compatible, MR Safe, Remote Center of Motion Needle-Guide Robot," in IEEE Transactions on Biomedical Engineering, vol. 65, no. 1, pp. 165-177, Jan. 2018. doi 10.1109/TBME.2017.2697766

[14] J. G. R. Bomers, D. G. H. Bosboom, G. H. Tigelaar, J. Sabisch, J. J. Fütterer, and D. Yakar, "Feasibility of a 2nd generation MR-compatible manipulator for transrectal prostate biopsy guidance," European Radiology, vol. 27, no. 4, pp. 1776-1782, 2017. doi: 10.1007/s00330-016$4504-2$ 